\title{
Transcriptome Analysis of Bovine Ovarian Follicles at Predeviation and Onset of Deviation Stages of a Follicular Wave
}

\author{
Pengfei Li, ${ }^{1}$ Jinzhu Meng, ${ }^{2}$ Wenzhong Liu, ${ }^{2}$ George W. Smith, ${ }^{3}$ Jianbo Yao, ${ }^{4}$ and Lihua Lyu ${ }^{2}$ \\ ${ }^{1}$ College of Life Science, Shanxi Agricultural University, Taigu, Shanxi 030801, China \\ ${ }^{2}$ College of Animal Science and Technology, Shanxi Agricultural University, Taigu, Shanxi 030801, China \\ ${ }^{3}$ Laboratory of Mammalian Reproductive Biology and Genomics, Departments of Animal Science and Physiology, \\ Michigan State University, East Lansing, MI 48824, USA \\ ${ }^{4}$ Division of Animal and Nutritional Sciences, West Virginia University, Morgantown, WV 26506, USA
}

Correspondence should be addressed to Lihua Lyu; adamlpf@163.com

Received 16 December 2015; Revised 28 February 2016; Accepted 29 February 2016

Academic Editor: Ferenc Olasz

Copyright (C) 2016 Pengfei Li et al. This is an open access article distributed under the Creative Commons Attribution License, which permits unrestricted use, distribution, and reproduction in any medium, provided the original work is properly cited.

For two libraries (PDF1 and ODF1) using Illumina sequencing 44,082,301 and 43,708,132 clean reads were obtained, respectively. After being mapped to the bovine RefSeq database, 15,533 genes were identified to be expressed in both types of follicles (cut-off RPKM > 0.5), of which 719 were highly expressed in bovine follicles (cut-off RPKM > 100). Furthermore, 83 genes were identified as being differentially expressed in ODF1 versus PDF1, where 42 genes were upregulated and 41 genes were downregulated. KEGG pathway analysis revealed two upregulated genes in ODF1 versus PDF1, CYP11A1, and CYP19A1, which are important genes in the steroid hormone biosynthesis pathway. This study represents the first investigation of transcriptome of bovine follicles at predeviation and onset of deviation stages and provides a foundation for future investigation of the regulatory mechanisms involved in follicular development in cattle.

\section{Introduction}

The ovarian follicle is an essential component of the reproductive process. It plays an important role in controlling the estrous cycle, determining estrous behaviour, ensuring oocyte competency and subsequent embryo survival rate, and determining both postovulation corpus luteum function and progesterone synthesis [1]. In a number of species, follicular growth is characterized by a wave-like pattern, with two or three waves occurring during the normal course of estrous cycles in cattle [2]. During each wave of follicular development, a cohort of antral follicles are induced to begin accelerated growth [3]. After a period of concurrent growth, a species specific number of follicles will then be selected to become dominant, while the remaining follicles will be lost through a process known as atresia. Diameter deviation is defined as the divergence in growth rates between the two largest follicles in a follicular wave [3]. The onset of diameter deviation occurs when the largest follicle reaches $8.5 \mathrm{~mm}$ in dairy cattle and marks initiation of divergence in growth rate and estradiol producing capacity between the F1 or largest (future dominant follicle) and F2 or second largest (future subordinate) growing follicles culminating the process of dominant follicle selection. While the exact mechanisms of dominant follicle selection are not completely understood, there have been many studies on the hormones and factors involved in follicular development. Antral follicles are dependent upon FSH for growth and each follicular wave is preceded by a transient rise in FSH concentrations [4]. Many growth factors linked to regulation of follicular development, such as inhibins, activins, and insulin-like growth factors and their binding proteins, have been identified in follicular fluid of individual bovine follicles [5]. These molecules can regulate follicular cell survival, proliferation, or death. Recent studies have attempted to understand the molecular regulation of follicular development in cattle [6,7]. However, the molecular mechanisms governing the wave-like pattern of follicular development are incompletely described, particularly at 
the onset of diameter deviation which is the first morphological indication of follicular dominance.

Traditionally, gene expression studies in the field of follicular development focus on the study of expression of candidate genes of interest. With the development of nextgeneration sequencing technologies, transcriptome profiling has become a powerful approach for identification of genes globally expressed in various tissues including ovarian follicles [8]. In the present study, we performed RNA-Seq of granulosa cell RNA from bovine ovarian follicles at predeviation (PD) and onset of deviation (OD) stages of a follicular wave in cattle to catalog the transcriptome and identify potential differentially expressed genes associated with these key stages of a follicular wave. This study provides a comprehensive sequence resource for future studies on follicular development in cattle.

\section{Materials and Methods}

2.1. Materials. All materials were obtained from SigmaAldrich (St. Louis, MO) unless otherwise stated.

2.2. Animal Model and Sample Collection. All animal procedures were approved by the Institutional Animal Care and Use Committee at Michigan State University. Estrus was synchronized in nonlactating Holstein dairy cows with two injections of prostaglandin F $2 \alpha$ (PGF $2 \alpha$; Prostamate; IVX Animal Health, St. Joseph, MO) administered 14 days apart, and follicular growth was observed and recorded by daily ultrasonography.

Ovaries were removed from cows at the following stages of the first follicular wave: predeviation (PD; approximately Day 3 after estrus; 1.5 days after emergence [emergence is the first scan where a new follicle at least $4 \mathrm{~mm}$ is detected by ovarian ultrasonography]) and onset of deviation (OD; first scan where growth of the F1 [largest; future dominant] follicle to $>8.5 \mathrm{~mm}$ was detected by ovarian ultrasonography). The F1 follicles were isolated from the PD and OD groups. Granulosa cells were isolated from the two types of follicles (PDF1 and ODF1), lysed, and stored at $-80^{\circ} \mathrm{C}$ immediately.

2.3. RNA Isolation. Total RNA was isolated from the lysed granulosa cells using the RNeasy mini kit (Qiagen) and DNase treated on column according to the manufacturer's protocol. The RNA integrity was evaluated by Agilient Bioanalyser and the RNA concentration was measured using a Nanodrop-1000 spectrophotometer. RNA samples with a RNA integrity number greater than 8 were selected for deep sequencing.

2.4. Library Preparation and Illumina Sequencing. RNA sequencing was performed by the WM Keck Center for Comparative and Functional Genomics at the University of Illinois at Urbana-Champaign. RNA samples from four follicles were pooled within each group (ODF1 or PDF1). RNA-Seq libraries were prepared with a TruSeq RNA Sample Preparation kit (Illumina) according to the manufacturer's instructions. The cDNA libraries were sequenced on one lane for 100 cycles using Illumina $\mathrm{HiSeq}^{\mathrm{TM}} 2000$ by a TruSeq SBS kit v5 (Illumina) and analyzed with pipeline version 1.8.

2.5. Identification of Differentially Expressed Genes and Pathway Analysis. The CLC Genomics Workbench (CLC bio, Aarhus, Denmark) was used to map the sequence reads to the bovine RefSeq database. The reads per kilobase per million reads (RPKM) values were calculated as the normalized transcript expression values [9]. A $Z$-test [10] was used to identify differentially expressed genes between ODF1 and PDF1 (FDR corrected $p$ value $<0.05$, RPKM cut-off $>0.5$, and RPKM fold change $>1.5$ ) using the CLC genomics workbench. DAVID software (https://david.ncifcrf.gov/gene2gene.jsp) was used to perform GO annotations and KEGG pathway analysis for highly expressed $(\mathrm{RPKM}>100)$ and differentially expressed genes.

\section{Results}

3.1. Illumina Sequencing. To identify differentially expressed genes involved in bovine follicular development, Illumina sequencing was used on two libraries constructed from RNA isolated from ODF1 and PDF1 follicles. After filtering, a total of 44,082,301 and 43,708,132 clean reads were obtained from PDF1 and ODF1 libraries, respectively. The clean reads were mapped to the bovine RefSeq database (containing 35,325 annotated transcripts). Using a cut-off value of RPKM > 0.5 , a total of 15,533 genes were identified in both types of follicles (Additional file 1: Table S1 in Supplementary Material available online at http://dx.doi.org/10.1155/2016/3472748), among which 719 are considered to be highly expressed (RPKM cut-off > 100) in bovine follicles (Additional file 2: Table S2).

3.2. GO Functional Classification and KEGG Pathway Analysis of Highly Expressed Genes. The top 30 highly expressed genes in granulosa cells of bovine follicles at the predeviation and onset of deviation stages are shown in Table 1. Many of them are known to be important for follicular growth and development, such as Serpin peptidase inhibitor clade E member 2 (SERPINE2), Inhibin alpha (INHA), Inhibin beta A (INHBA), and Follistatin (FST). GO functional classification of these highly expressed genes was performed using DAVID software. All 719 highly expressed genes can be assigned into 22 groups under three categories (biological process, 39\%; cellular component, 44\%; and molecular function, 17\%) based on their putative functions (Figure 1). Many of the highly expressed genes are involved in metabolic process, multicellular organismal process, and binding. KEGG pathway analysis showed that the highly expressed genes are involved in 12 major pathways (Figure 2), of which the most significantly enriched genes are involved in ribosome pathway.

3.3. Differentially Expressed Genes in ODF1 versus PDF1. Using RPKM cut-off $>0.5$ and fold change cut-off $>1.5$ at FDR corrected $p$ value $<0.05$, a total of 83 differentially expressed genes were identified, with 41 downregulated genes (Table 2) 

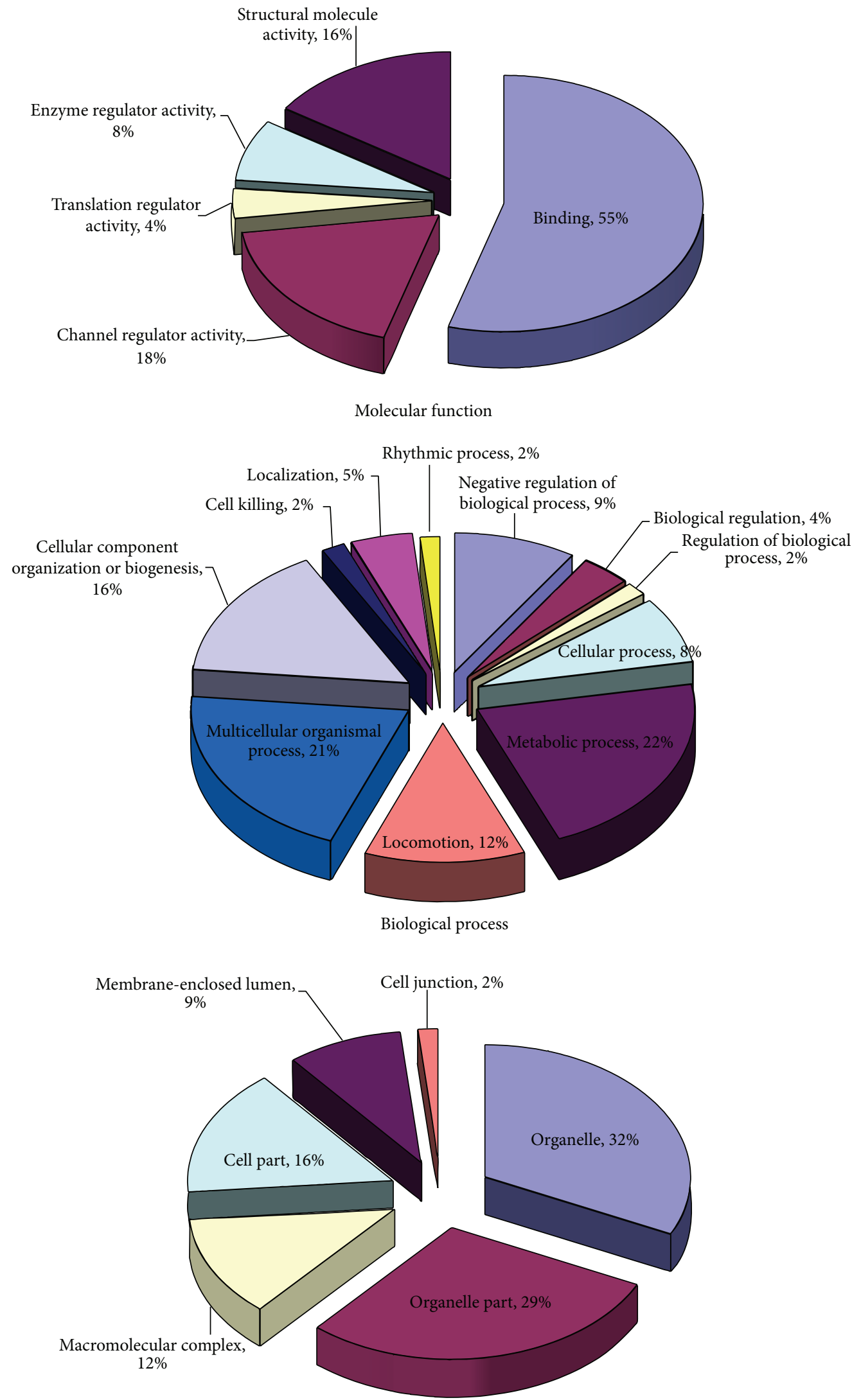

Cellular component

FIGURE 1: Highly expressed genes GO analysis. 
TABLE 1: Top 30 highly expressed genes in ODF1 and PDF1 follicles.

\begin{tabular}{|c|c|c|}
\hline Gene name & PDF1-RPKM & ODF1-RPKM \\
\hline Glutathione S-transferase alpha 3 & 12445.32 & 17611.44 \\
\hline Serpin peptidase inhibitor clade E member 2 & 6816.847 & 11075.44 \\
\hline Inhibin alpha & 6341.615 & 9658.832 \\
\hline Inhibin beta $\mathrm{A}$ & 5824.462 & 9440.244 \\
\hline Serglycin & 8808.27 & 7315.526 \\
\hline Follistatin & 3435.171 & 4998.088 \\
\hline Cytochrome c oxidase subunit I-like & 5017.207 & 3707.555 \\
\hline Unknown & 7026.759 & 3115.129 \\
\hline Cytochrome c oxidase subunit III-like & 3508.943 & 2440.522 \\
\hline Cytochrome c oxidase subunit I-like & 3252.12 & 2372.119 \\
\hline Unknown & 5741.943 & 2262.251 \\
\hline Milk fat globule-EGF factor 8 protein & 2439.639 & 2077.699 \\
\hline Vimentin & 2032.584 & 2050.732 \\
\hline Lysosomal protein transmembrane 4 beta & 1539.453 & 2025.655 \\
\hline Gap junction protein alpha 1 & 1408.231 & 1975.614 \\
\hline Cytochrome P450 family 19 subfamily A polypeptide 1 & 656.8769 & 1944.887 \\
\hline Eukaryotic translation elongation factor 1 alpha 1 & 1864.128 & 1924.407 \\
\hline Low density lipoprotein receptor-related protein 8 apolipoprotein $\mathrm{E}$ receptor & 1061.542 & 1897.644 \\
\hline Enolase 1 & 1005.447 & 1707.612 \\
\hline Heat shock protein 8 & 1615.28 & 1700.711 \\
\hline Ribosomal protein L18a & 1559.355 & 1680.399 \\
\hline Glyceraldehydes 3 phosphate dehydrogenase & 1342.963 & 1670.066 \\
\hline Ribosomal protein S27a & 1576.131 & 1589.545 \\
\hline Ribosomal protein & 1627.751 & 1566.558 \\
\hline ST3 beta-galactoside alpha-2,3-sialyltransferase 4 & 1096.954 & 1542.973 \\
\hline Ribosomal protein L4 & 1443.707 & 1502.241 \\
\hline Ribosomal protein S8 & 1563.222 & 1490.917 \\
\hline Tribbles homolog 2 & 1411.494 & 1461.935 \\
\hline Ribosomal protein S3A & 1409.527 & 1450.511 \\
\hline Cytochrome P450, family 11, subfamily A, polypeptide 1 & 908.9737 & 1425.869 \\
\hline
\end{tabular}

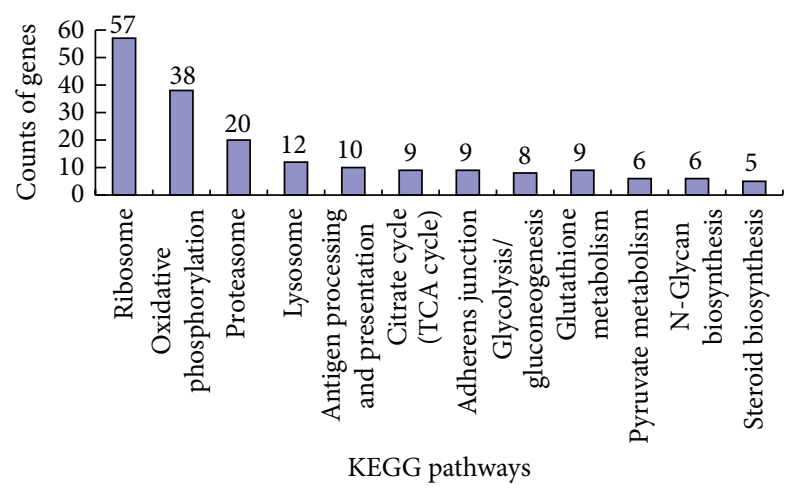

FIGURE 2: Highly expressed genes KEGG pathways analysis.

and 42 upregulated genes (Table 3 ) in ODF1 versus PDF1. To understand the functions of these differentially expressed genes, GO analysis was performed. The upregulated genes were categorized into 14 functional groups under 3 major GO classifications: biological process (35\%), cellular component
(30\%), and molecular function (35\%) (Table 4). Many of the differentially expressed genes are known to play a role in ovarian follicular development (Table 5). For example, serine protease 23 (PRSS23) is expressed in granulosa cells and may play a crucial role in follicular atresia, whereas serine protease 35 (PRSS35) is also expressed in granulosa cells and may be involved in ovulation and CL formation and regression. KEGG pathway analysis of the upregulated genes demonstrated that two important genes (CYP11A1 and CYP19A1) in the steroid hormone biosynthesis pathway are upregulated in ODF1 versus PDF1.

\section{Discussion}

Follicular growth occurs in a characteristic wave-like pattern in monotocous species such as cattle $[3,5,11]$. A transient increase in FSH triggers initiation of each follicular wave $[5,11,12]$. Emergence is defined as the first day a new follicle $>4 \mathrm{~mm}$ in diameter is detected and is the first chronological event marking a new follicular wave that is detectable by 
TABLE 2: List of downregulated genes in ODF1 versus PDF1 and their functions.

\begin{tabular}{|c|c|c|c|c|c|c|}
\hline Gene symbol & GenBank number & $\begin{array}{l}\text { PDF1 } \\
\text { RPKM }\end{array}$ & $\begin{array}{l}\text { ODF1 } \\
\text { RPKM } \\
\end{array}$ & $\begin{array}{l}\text { Fold } \\
\text { change }\end{array}$ & $\begin{array}{c}\text { FDR corrected } \\
p \text { value }\end{array}$ & Gene product functions \\
\hline ACTR1A & NM_001193248.1 & 17.31 & 0.43 & -39.45 & $2.63 \times 10^{-2}$ & Vesicle motility \\
\hline LOC787803 & XM_002700116.1 & 45.66 & 2.15 & -20.79 & $3.41 \times 10^{-7}$ & Unknown \\
\hline PPP1R14A & XM_002694966.1 & 68.29 & 3.99 & -16.76 & $7.75 \times 10^{-11}$ & Protein phosphatase inhibitor \\
\hline OLA1 & NM_001046045.1 & 22.71 & 1.74 & -12.79 & $1.10 \times 10^{-2}$ & $\begin{array}{l}\text { Hydrolase activity and GTP } \\
\text { binding }\end{array}$ \\
\hline QRFPR & NM_001192681.1 & 19.15 & 1.55 & -12.07 & $4.40 \times 10^{-2}$ & Modulate adenylate cyclase \\
\hline RMRP & NR_036646.1 & 149.79 & 13.57 & -10.82 & & Lncrna class \\
\hline RN5-8S1 & NR_036643.1 & 9330.16 & 856.09 & -10.68 & & Unknown \\
\hline LOC100335749 & XR_083021.1 & 35.26 & 4.58 & -7.54 & $8.45 \times 10^{-4}$ & $\begin{array}{l}\text { Senescence-associated } \\
\text { protein-like }\end{array}$ \\
\hline $\mathrm{C} 11 \mathrm{H} 2$ orf 40 & NM_001038113.1 & 41.02 & 5.84 & -6.88 & $2.22 \times 10^{-4}$ & Esophageal cancer \\
\hline BOLA & NM_001040532.1 & 74.93 & 11.19 & -6.56 & $1.01 \times 10^{-8}$ & Transcription \\
\hline ANGPT2 & NM_001098855.1 & 39.04 & 7.91 & -4.83 & $3.56 \times 10^{-3}$ & Angiogenic signal \\
\hline VNN1 & NM_001024556.2 & 33.51 & 7.26 & -4.52 & $1.94 \times 10^{-2}$ & Amidohydrolase \\
\hline KRT2 & XM_001254015.1 & 40.64 & 9.41 & -4.23 & $6.20 \times 10^{-3}$ & Keratinocyte activation \\
\hline $\mathrm{IHH}$ & NM_001076870.2 & 42.84 & 9.98 & -4.21 & $3.93 \times 10^{-3}$ & Smoothened \\
\hline RN18S1 & NR_036642.1 & 4431.16 & 1040.31 & -4.17 & & Unknown \\
\hline BOLA & NM_001038518.1 & 101.2 & 26.48 & -3.74 & $6.16 \times 10^{-8}$ & Transcription \\
\hline LOC100335409 & XM_002705970.1 & 1012.71 & 275.19 & -3.61 & & Unknown \\
\hline LOC100140002 & XR_084188.1 & 38.53 & 10.81 & -3.49 & $3.63 \times 10^{-2}$ & Envelope glycoprotein-like \\
\hline $\mathrm{ADM}$ & NM_173888.3 & 203.61 & 61 & -3.27 & & Adrenomedullin \\
\hline 4-Sep & NM_001034651.1 & 69.77 & 22.91 & -2.98 & $9.40 \times 10^{-4}$ & Cytokinesis, platelet secretion \\
\hline ITPR1 & NM_174841.2 & 61.79 & 22.18 & -2.73 & $9.78 \times 10^{-3}$ & Intracellular channel \\
\hline RN28S1 & NR_036644.1 & 537.61 & 205.32 & -2.56 & & Unknown \\
\hline LOC100336997 & XM_002685421.1 & 5741.94 & 2262.25 & -2.49 & & Unknown \\
\hline PRSS35 & NM_001035457.3 & 134.05 & 55.07 & -2.38 & $1.21 \times 10^{-5}$ & $\begin{array}{l}\text { Ovulation, CL formation and } \\
\text { regression }\end{array}$ \\
\hline LOC100337434 & XR_083937.1 & 7026.76 & 3115.13 & -2.21 & & Unknown \\
\hline LOC100140226 & XM_001787664.2 & 1183.02 & 532.17 & -2.18 & & Zinc finger protein 347 -like \\
\hline LOC100337402 & XR_083935.1 & 908.3 & 413.36 & -2.15 & & Unknown \\
\hline LOC511901 & XM_589328.5 & 102.29 & 49.83 & -2.01 & $1.42 \times 10^{-2}$ & H1 histone \\
\hline LOC100137883 & XM_002706880.1 & 98.48 & 49.64 & -1.94 & $3.47 \times 10^{-2}$ & Thymosin beta-4-like \\
\hline $\mathrm{CDH} 2$ & NM_001166492.1 & 103.68 & 53.3 & -1.91 & $3.44 \times 10^{-2}$ & Neuronal recognition \\
\hline APOA1 & NM_174242.3 & 126.56 & 66.22 & -1.87 & $1.07 \times 10^{-2}$ & $\begin{array}{l}\text { Activates spermatozoa } \\
\text { motility }\end{array}$ \\
\hline PAPSS2 & NM_001076075.1 & 119.75 & 65.23 & -1.8 & $3.68 \times 10^{-2}$ & Skeletogenesis \\
\hline LOC100299201 & XR_084007.1 & 327.62 & 178.8 & -1.79 & $1.28 \times 10^{-7}$ & Ribosomal protein \\
\hline GSTA5 & NM_001099016.1 & 138.14 & 80.02 & -1.69 & $4.93 \times 10^{-2}$ & Glutathione transferase \\
\hline AKR1B1 & NM_001012519.1 & 182.74 & 108.97 & -1.64 & $1.33 \times 10^{-2}$ & Electron carrier activity \\
\hline SLCO1A2 & NM_174654.2 & 192.47 & 119.34 & -1.58 & $2.65 \times 10^{-2}$ & Mediates transport \\
\hline HERC1 & NM_001103282.1 & 222.29 & 138.46 & -1.57 & $1.01 \times 10^{-2}$ & Membrane trafficking \\
\hline CWC25 & NM_001105359.1 & 433.81 & 274.72 & -1.55 & $7.12 \times 10^{-6}$ & $\begin{array}{l}\text { Alternatively spliced } \\
\text { transcripts }\end{array}$ \\
\hline ACOT11 & NM_001103275.1 & 693.81 & 440.12 & -1.54 & $6.15 \times 10^{-10}$ & Acyl-Coa thioesterase activity \\
\hline LOC615589 & NM_001098467.1 & 245.33 & 157.07 & -1.53 & $1.10 \times 10^{-2}$ & Keratin-like protein \\
\hline C12H13orf18 & NM_001102041.1 & 269.27 & 173.55 & -1.52 & $6.34 \times 10^{-3}$ & Unknown \\
\hline
\end{tabular}


TABLE 3: List of upregulated genes in ODF1 versus PDF1 and their functions.

\begin{tabular}{|c|c|c|c|c|c|c|}
\hline Gene symbol & GenBank number & $\begin{array}{l}\text { PDF1 } \\
\text { RPKM }\end{array}$ & $\begin{array}{l}\text { ODF1 } \\
\text { RPKM }\end{array}$ & $\begin{array}{l}\text { Fold } \\
\text { change }\end{array}$ & $\begin{array}{l}\text { FDR corrected } \\
p \text { value }\end{array}$ & Gene product functions \\
\hline GAPDH & XM_001252511.3 & 1.91 & 59.65 & 31.91 & $9.83 \times 10^{-11}$ & Microtubule and NAD binding \\
\hline BOLA-N & NM_001105651.1 & 14.28 & 174.88 & 12.50 & & Unknown \\
\hline PPP1R14A & NM_001193070.1 & 6.06 & 65.75 & 11.08 & $8.98 \times 10^{-10}$ & Smooth muscle contraction \\
\hline LOC505676 & NM_001193296.1 & 14.04 & 111.19 & 8.09 & & Unknown \\
\hline LOC100337308 & XM_002684003.1 & 3.72 & 23.27 & 6.39 & $4.40 \times 10^{-2}$ & Unknown \\
\hline MT1A & NM_001040492.2 & 26.50 & 150.75 & 5.81 & & Bind heavy metals \\
\hline LOC100125916 & NM_001105487.1 & 14.80 & 83.88 & 5.78 & $1.51 \times 10^{-9}$ & Unknown \\
\hline TNFAIP6 & NM_001007813.1 & 11.17 & 46.61 & 4.26 & $1.06 \times 10^{-3}$ & $\begin{array}{l}\text { Cell-cell and cell-matrix } \\
\text { interactions }\end{array}$ \\
\hline BEX2 & NM_001077034.1 & 93.97 & 345.00 & 3.75 & & Mitochondrial apoptosis \\
\hline GPR85 & NM_001075150.2 & 10.06 & 35.54 & 3.61 & $4.05 \times 10^{-2}$ & G-protein coupled receptor \\
\hline PPM1K & NM_001046474.1 & 34.75 & 108.32 & 3.18 & $2.52 \times 10^{-7}$ & Cellular survival and development \\
\hline CYP19A1 & NM_174305.1 & 656.88 & 1944.89 & 3.02 & & Estrogen biosynthesis \\
\hline MT1E & NM_001114857.1 & 54.11 & 152.16 & 2.87 & $2.61 \times 10^{-9}$ & Bind heavy metals \\
\hline ETNK2 & XM_002693881.1 & 22.35 & 61.96 & 2.83 & $4.65 \times 10^{-3}$ & Ethanolamine phosphorylation \\
\hline CHST11 & NM_001192668.1 & 57.39 & 154.72 & 2.75 & $6.77 \times 10^{-9}$ & Biosynthesis chondroitin sulfate \\
\hline MT2A & NM_001075140.1 & 49.37 & 130.49 & 2.70 & $4.16 \times 10^{-7}$ & Bind heavy metals \\
\hline PRSS23 & NM_001080306.1 & 58.01 & 151.79 & 2.67 & $2.74 \times 10^{-8}$ & Follicular atresia \\
\hline TXNIP & NM_001101875.2 & 98.64 & 231.47 & 2.40 & $6.64 \times 10^{-11}$ & Oxidative stress mediator \\
\hline NPR3 & NM_174127.2 & 47.55 & 106.35 & 2.28 & $5.93 \times 10^{-4}$ & $\begin{array}{l}\text { Natriuretic peptide hormone } \\
\text { receptor }\end{array}$ \\
\hline GREB1 & NM_001205631.1 & 35.17 & 78.20 & 2.27 & $1.30 \times 10^{-2}$ & $\begin{array}{l}\text { Estrogen-stimulated cell } \\
\text { proliferation }\end{array}$ \\
\hline EIF4EBP1 & NM_001077893.1 & 138.11 & 294.07 & 2.17 & $1.30 \times 10^{-11}$ & $\begin{array}{l}\text { Mediates protein translation } \\
\text { regulation }\end{array}$ \\
\hline PIK3R1 & NM_174575.1 & 113.42 & 210.95 & 1.90 & $1.21 \times 10^{-5}$ & Insulin actions metabolic \\
\hline LRP8 & NM_001097565.1 & 1061.54 & 1897.64 & 1.82 & & Sperm maturation \\
\hline SCD5 & NM_001076945.1 & 124.08 & 210.97 & 1.74 & $4.25 \times 10^{-4}$ & Energy metabolism \\
\hline ENO1 & NM_174049.2 & 1005.45 & 1707.61 & 1.73 & & Tumor suppressor \\
\hline LDHA & NM_174099.2 & 221.85 & 366.73 & 1.69 & $3.41 \times 10^{-7}$ & Affiliated with lncrna \\
\hline SERPINE2 & NM_174669.2 & 6816.85 & 11075.44 & 1.66 & & Serine protease inhibitor \\
\hline INHBA & NM_174363.2 & 5824.46 & 9440.24 & 1.65 & & $\begin{array}{l}\text { Regulate gonadal stromal cell } \\
\text { proliferation }\end{array}$ \\
\hline TMEM176B & NM_001099145.1 & 106.45 & 170.39 & 1.63 & $2.16 \times 10^{-2}$ & Dendritic cells maturation \\
\hline OAT & NM_001034240.1 & 395.42 & 630.73 & 1.63 & $1.71 \times 10^{-11}$ & Ornithine aminotransferase \\
\hline CYP11A1 & NM_176644.2 & 908.97 & 1425.87 & 1.60 & & Cholesterol to pregnenolone \\
\hline OBSL1 & XM_002685586.1 & 221.33 & 338.40 & 1.56 & $1.16 \times 10^{-4}$ & Regulate ubiquitin ligase complex \\
\hline ARFGAP3 & NM_001075974.1 & 273.92 & 418.22 & 1.56 & $5.26 \times 10^{-6}$ & Gtpase-activating protein \\
\hline ITGB5 & NM_174679.2 & 153.24 & 233.56 & 1.56 & $7.68 \times 10^{-3}$ & Fibronectin receptor \\
\hline INHA & NM_174094.3 & 6341.61 & 9658.83 & 1.55 & & Gonadal hormone secretion \\
\hline OPTN & NM_001034602.1 & 160.13 & 243.40 & 1.55 & $5.92 \times 10^{-3}$ & Affect cell death \\
\hline PTGR1 & NM_001035281.1 & 200.07 & 301.66 & 1.54 & $9.40 \times 10^{-4}$ & $\begin{array}{l}\text { Inactivation of the chemotactic } \\
\text { factor }\end{array}$ \\
\hline STBD1 & XM_002688357.1 & 143.24 & 215.66 & 1.54 & $2.15 \times 10^{-2}$ & Bind to carbohydrates \\
\hline LOC532189 & XR_083049.1 & 340.18 & 510.18 & 1.53 & $5.69 \times 10^{-7}$ & Carboxypeptidase \\
\hline TMEM20 & NM_001076470.1 & 191.10 & 286.48 & 1.53 & $2.10 \times 10^{-3}$ & Solute carrier \\
\hline ECE1 & NM_181009.2 & 461.24 & 691.33 & 1.53 & $8.98 \times 10^{-10}$ & $\begin{array}{l}\text { Converts big endothelin-1 to } \\
\text { endothelin-1 }\end{array}$ \\
\hline GNG10 & NM_001114512.1 & 617.73 & 918.26 & 1.52 & $7.59 \times 10^{-13}$ & Signal transducer \\
\hline
\end{tabular}


TABLE 4: GO analysis of genes upregulated in ODF1 versus PDF1.

\begin{tabular}{lll}
\hline Category & Term & Genes \\
\hline Biological process & Hemoglobin biosynthetic process & INHBA, INHA \\
Biological process & Antigen processing and presentation & LOC505676, LOC100125916, BOLA-N \\
Biological process & Insulin receptor signaling pathway & EIF4EBP1, PIK3R1 \\
Biological process & Antigen processing and presentation of peptide antigen & LOC100125916, BOLA-N \\
Biological process & Response to insulin stimulus & EIF4EBP1, PIK3R1 \\
Biological process & Cellular hormone metabolic process & ECE1, CYP11A1 \\
Biological process & Cellular response to hormone stimulus & EIF4EBP1, PIK3R1 \\
Biological process & Regulation of myeloid cell differentiation & INHBA, PIK3R1 \\
Cellular component & Inhibin complex & INHBA, INHA \\
Cellular component & MHC protein complex & LOC505676, LOC100125916, BOLA-N \\
Cellular component & Plasma membrane & LOC505676, ARFGAP3, ECE1, GNG10, ITGB5, INHA, \\
Molecular function & Metal ion binding & LOC100125916, BOLA-N, ENO1 \\
Molecular function & Peptidase activity & ARFGAP3, PTGR1, ECE1, MT1A, CYP11A1, PPM1K, \\
Molecular function & Iron ion binding & LRP8, SCD5, CYP19A1, ENO1 \\
\hline
\end{tabular}

TABLE 5: Differentially expressed genes between ODF1 and PDF1 with known or predicted roles in follicular development.

\begin{tabular}{lcccl}
\hline Gene symbol & Fold change & $\begin{array}{c}\text { FDR corrected } \\
p \text { value }\end{array}$ & $\begin{array}{c}\text { Up- or downregulation } \\
\text { in ODF1/PDF1 }\end{array}$ & Gene product functions \\
\hline PPM1K & 3.18 & $2.52 \times 10^{-7}$ & Upregulation & Cellular survival and development \\
BEX2 & 3.75 & & Upregulation & Mitochondrial apoptosis \\
CYP19A1 & 3.02 & & Upregulation & Estrogen biosynthesis \\
PRSS23 & 2.67 & $2.74 \times 10^{-8}$ & Upregulation & Follicular atresia \\
GREB1 & 2.27 & $1.30 \times 10^{-2}$ & Upregulation & Estrogen-stimulated cell proliferation \\
SERPINE2 & 1.66 & & Upregulation & Serine protease inhibitor \\
INHBA & 1.65 & & Upregulation & Regulate gonadal stromal cell proliferation \\
CYP11A1 & 1.60 & & Upregulation & Cholesterol to pregnenolone \\
INHA & 1.55 & Upregulation & Cenadal hormone secretion \\
TNFAIP6 & 4.26 & $1.06 \times 10^{-3}$ & Upregulation & Affect cell death \\
OPTN & 1.55 & $5.92 \times 10^{-3}$ & Downregulation & Ovulation and CL formation and regression \\
PRSS35 & -2.38 & $1.21 \times 10^{-5}$ & Downregulation & Activates spermatozoa motility \\
APOA1 & -1.87 & $1.07 \times 10^{-2}$ & Downregulation & Glutathione transferase \\
GSTA5 & -1.69 & $4.93 \times 10^{-2}$ & &
\end{tabular}

ultrasonography. After emergence, follicles in the cohort initially grow at a similar rate (common growth phase) prior to deviation [3]. However, the molecular mechanisms involved regulating the onset of deviation are not well understood, in order to characterize the differences in gene expression that associated with follicular development in different follicles sized in diameter, which the previous studies examined using microarray technology [13-17]. To further investigate the bovine granulosa cell transcriptome and molecular alterations associated with onset of deviation, we examined the transcriptome at specific stages of the estrous cycle.

Illumina sequencing technology was used to determine gene expression levels in ODF1 and PDF1 follicles. A total of 15,533 genes were identified in both types of follicles and 83 of them were identified as differentially expressed between
ODF1 and PDF1. Our study provided novel information on the bovine granulosa cell transcriptome and identified specific transcripts highly expressed in granulosa cells of bovine follicles prior to and at onset of deviation, including transcripts encoding for several housekeeping genes (e.g., ribosomal proteins L18a, S27a, and L4) and genes with wellestablished roles in regulation of ovarian function (e.g., INHBA, INHBB, and FST). Of particular interest was SERPINE2, which is abundantly expressed in granulosa cells of follicles collected at both the predeviation and onset of deviation stages of a follicular wave, illustrating its potential importance in bovine ovarian follicular development. Estradiol and SERPINE2 secretion are positively correlated, but estradiol treatment cannot alter the expression of SERPINE2. $\mathrm{FSH}$ and growth factors can directly regulate the expression 
and secretion of SERPINE2 in granulosa cells, and SERPINE2 is an antiapoptotic factor, which may regulate atresia in bovine follicles [18]. Eleven SERPINE genes are expressed in bovine follicles, but only SERPINE2, SERPINE5, and SERPINE6 are expressed in the granulosa cells [19].

KEGG analysis revealed upregulated genes associated with onset of deviation (CYP11A1 and CYP19A1) involved in the steroid hormone biosynthesis pathways that play an essential role during follicular development. Proteins encoded by CYP11A1 and CYP19A1 genes are members of the cytochrome P450 superfamily, which are monooxygenases that catalyze many reactions involved in steroidogenesis. Previous studies suggested that CYP19A1 was regulated by multiple pathways, including estrogen receptors and CAMP/ protein kinase A which are activated by FSHR in granulosa cells, and these regulatory mechanisms are likely critical for acquisition of follicular dominance in cattle [20]. Our transcriptome sequencing data is consistent with these results. The increase in transcript abundance for CYP19A1 in ODF1 versus PDF1 follicles is consistent with the increase in estradiol producing capacity associated with diameter deviation [3].

It is acknowledged that study design was not optimal due to limited biological replication because single pooled samples ( $n=4$ per group) were used in Illumina sequencing analysis. Despite such limitations, results have significantly enhanced understanding of bovine follicle transcriptome composition and potential differences in gene expression associated with follicular development that are foundational to further study in the future; several interesting candidates were revealed for future investigation, particularly genes linked to regulation of cell proliferation and survival. For example, results of present studies suggest that PPM1K, a $\mathrm{Mn}^{2+} / \mathrm{Mg}^{2+}$-dependent protein phosphatase of PPM family, is potentially upregulated with the onset of deviation in the granulosa cell layer of bovine follicles. This protein is critical for cell survival and embryonic development and can regulate the mitochondrial membrane permeability transition pore opening [21]. Potential upregulation of granulosa cell BEX2 and GREB1 transcript abundance was also noted in association with onset of dominance and may be associated with enhanced granulosa cell survival. BEX2 can downregulate apoptosis and activate the JNK (Jun NH2-terminal kinase) pathway, and these effects can be abolished by administration of a JNK specific inhibitor [22]. GREB1 is an estrogen receptor and coactivator linked to cell proliferation and GREB1 expression is estrogen dependent. It is possible that increased expression of PPM1K, BEX2, and GREB1 may be associated with granulosa cell proliferation and survival during the onset of deviation [23]. TNFAIP6/TSG6 is tumor necrosis factor and alpha-induced protein 6 ; it is suggested that TSG6 plays a role in cell-cell or cell-cell matrix interactions during inflammation and tumorigenesis. High LH/hCGR gene expression intensity was associated with TNFAIP6/TSG6 gene expression which has a pivotal importance in the mucification of the COC during the preovulatory period [24]. It is suggested that the expression levels of TNFAIP6/TSG6 were nearly 280 -fold in granulosa cells of large follicles than that of small follicles [14]. In our study, TNFAIP6/TSG6 was also differentially expressed in ODF1 and PDF1 with a 4.26-fold change. Altogether, these characteristics suggest that TNFAIP6/TSG6 plays a crucial role in accelerating follicle growth during follicular waves in cattle.

\section{Conclusions}

The present study characterized the granulosa cell transcriptome of bovine follicles at specific stages of follicular development and identified 83 differentially expressed genes potentially associated with onset of deviation, many of which are linked to regulation of follicular development. The study provides a foundation for future studies to investigate regulation of granulosa cell expressed genes and the regulatory mechanisms controlling antral follicle development during follicular waves in cattle.

\section{Competing Interests}

The authors declare that they have no competing interests.

\section{Acknowledgments}

This study was supported by the Chinese Natural Science Foundation (Grant no. 31172211), Introduction of Advanced International Agricultural Science and Technology Plan Project Fund (Grant no. 2010-Z43) to Lihua Lyu, Shanxi Province Science and Technology Research Project (Grant no. 20130311027-2) and Shanxi Agriculture University Introduction of Doctor Scientific Research Startup Fund (Grant no. 2014ZZ04) to Pengfei Li. Agriculture, and Food Research Initiative Competitive Grant no. 2009-65203-05700 from the USDA National Institute of Food and Agriculture to George W. Smith and by Michigan AgBioResearch (George W. Smith). The authors thank Liying Qiao and Wenge $\mathrm{Hu}$, technicians of the College of Animal Science and Technology, Shanxi Agricultural University, for their technical assistance.

\section{References}

[1] M. G. Diskin, D. R. Mackey, J. F. Roche, and J. M. Sreenan, "Effects of nutrition and metabolic status on circulating hormones and ovarian follicle development in cattle," Animal Reproduction Science, vol. 78, no. 3-4, pp. 345-370, 2003.

[2] A. C. O. Evans, "Characteristics of ovarian follicle development in domestic animals," Reproduction in Domestic Animals, vol. 38, no. 4, pp. 240-246, 2003.

[3] M. A. Beg and O. J. Ginther, "Follicle selection in cattle and horses: role of intrafollicular factors," Reproduction, vol. 132, no. 3, pp. 365-377, 2006.

[4] A. C. O. Evans and M. J. Canty, "Physiology of follicle development in cattle," in Proceedings of the WBC Congress, vol. 23, pp. 11-16, 2004.

[5] J. J. Ireland, M. Mihm, E. Austin, M. G. Diskin, and J. F. Roche, "Historical perspective of turnover of dominant follicles during the bovine estrous cycle: key concepts, studies, advancements, and terms," Journal of Dairy Science, vol. 83, no. 7, pp. 1648-1658, 2000 . 
[6] A. C. O. Evans, J. L. H. Ireland, M. E. Winn et al., "Identification of genes involved in apoptosis and dominant follicle development during follicular waves in cattle," Biology of Reproduction, vol. 70, no. 5, pp. 1475-1484, 2004.

[7] T. Fayad, V. Lévesque, J. Sirois, D. W. Silversides, and J. G. Lussier, "Gene expression profiling of differentially expressed genes in granulosa cells of bovine dominant follicles using suppression subtractive hybridization," Biology of Reproduction, vol. 70, no. 2, pp. 523-533, 2004.

[8] L. Lv, F. Jimenez-Krassel, A. Sen et al., "Evidence supporting a role for cocaine- and amphetamine-regulated transcript (CARTPT) in control of granulosa cell estradiol production associated with dominant follicle selection in cattle," Biology of Reproduction, vol. 81, no. 3, pp. 580-586, 2009.

[9] A. Mortazavi, B. A. Williams, K. McCue, L. Schaeffer, and B. Wold, "Mapping and quantifying mammalian transcriptomes by RNA-Seq," Nature Methods, vol. 5, no. 7, pp. 621-628, 2008.

[10] A. J. Kal, A. J. van Zonneveld, V. Benes et al., "Dynamics of gene expression revealed by comparison of serial analysis of gene expression transcript profiles from yeast grown on two different carbon sources," Molecular Biology of the Cell, vol. 10, no. 6, pp. 1859-1872, 1999.

[11] J. E. Fortune, G. M. Rivera, A. C. O. Evans, and A. M. Turzillo, "Differentiation of dominant versus subordinate follicles in cattle," Biology of Reproduction, vol. 65, no. 3, pp. 648-654, 2001.

[12] J. E. Fortune, "Ovarian follicular growth and development in mammals," Biology of Reproduction, vol. 50, no. 2, pp. 225-232, 1994.

[13] L. Nemcova, D. Jansova, K. Vodickova-Kepkova et al., "Detection of genes associated with developmental competence of bovine oocytes," Animal Reproduction Science, vol. 166, pp. 5871, 2016.

[14] N. Hatzirodos, H. F. Irving-Rodgers, K. Hummitzsch, M. L. Harland, S. E. Morris, and R. J. Rodgers, "Transcriptome profiling of granulosa cells of bovine ovarian follicles during growth from small to large antral sizes," BMC Genomics, vol. 15, article 24, 2014.

[15] R. Romar, T. De Santis, P. Papillier et al., "Expression of maternal transcripts during bovine oocyte in vitro maturation is affected by donor age," Reproduction in Domestic Animals, vol. 46, no. 1, pp. e23-e30, 2011.

[16] P. L. Pfeffer, B. Sisco, M. Donnison, J. Somers, and C. Smith, "Isolation of genes associated with developmental competency of bovine oocytes," Theriogenology, vol. 68, no. 1, pp. S84-S90, 2007.

[17] N. Ghanem, M. Hölker, F. Rings et al., "Alterations in transcript abundance of bovine oocytes recovered at growth and dominance phases of the first follicular wave," BMC Developmental Biology, vol. 7, article 90, 2007.

[18] M. Cao, E. Nicola, V. M. Portela, and C. A. Price, "Regulation of serine protease inhibitor-E2 and plasminogen activator expression and secretion by follicle stimulating hormone and growth factors in non-luteinizing bovine granulosa cells in vitro," Matrix Biology, vol. 25, no. 6, pp. 342-354, 2006.

[19] K.-G. Hayashi, K. Ushizawa, M. Hosoe, and T. Takahashi, "Differential gene expression of serine protease inhibitors in bovine ovarian follicle: possible involvement in follicular growth and atresia," Reproductive Biology and Endocrinology, vol. 9, article 72,2011

[20] W. Luo and M. C. Wiltbank, "Distinct regulation by steroids of messenger RNAs for FSHR and CYP19A1 in bovine granulosa cells," Biology of Reproduction, vol. 75, pp. 217-225, 2006.
[21] G. Lu, S. Ren, P. Korge et al., "A novel mitochondrial matrix serine/threonine protein phosphatase regulates the mitochondria permeability transition pore and is essential for cellular survival and development," Genes and Development, vol. 21, no. 7, pp. 784-796, 2007.

[22] X. Zhou, Q. Meng, X. Xu et al., "Bex2 regulates cell proliferation and apoptosis in malignant glioma cells via the c-Jun NH2terminal kinase pathway," Biochemical and Biophysical Research Communications, vol. 427, no. 3, pp. 574-580, 2012.

[23] L. A. Laviolette, K. M. Hodgkinson, N. Minhas, C. PerezIratxeta, and B. C. Vanderhyden, " $17 \beta$-estradiol upregulates GREB1 and accelerates ovarian tumor progression in vivo," International Journal of Cancer, vol. 135, no. 5, pp. 1072-1084, 2014.

[24] D. Haouzi, S. Assou, K. Mahmoud et al., "LH/hCGR gene expression in human cumulus cells is linked to the expression of the extracellular matrix modifying gene TNFAIP6 and to serum estradiol levels on day of hCG administration," Human Reproduction, vol. 24, no. 11, pp. 2868-2878, 2009. 

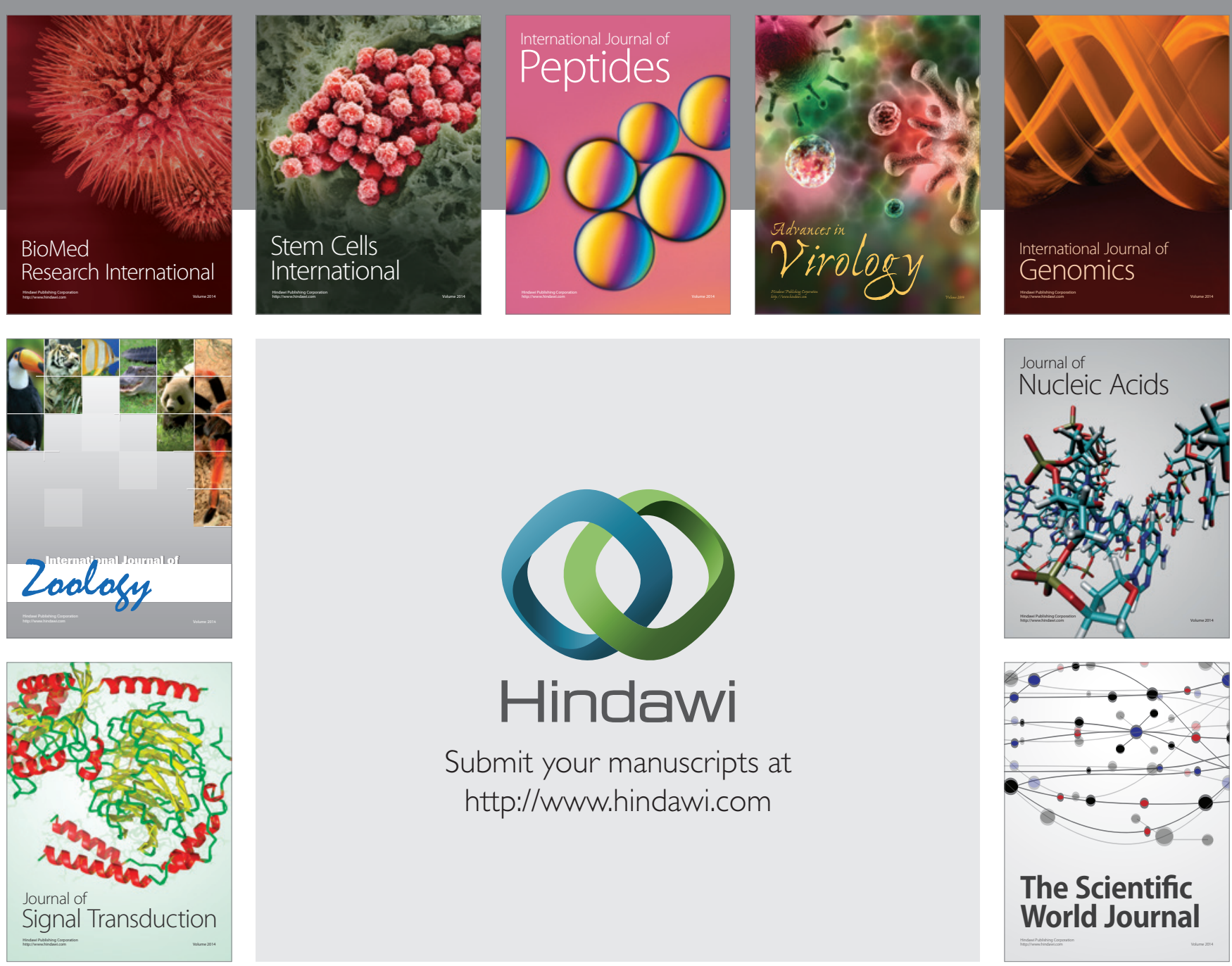

Submit your manuscripts at

http://www.hindawi.com
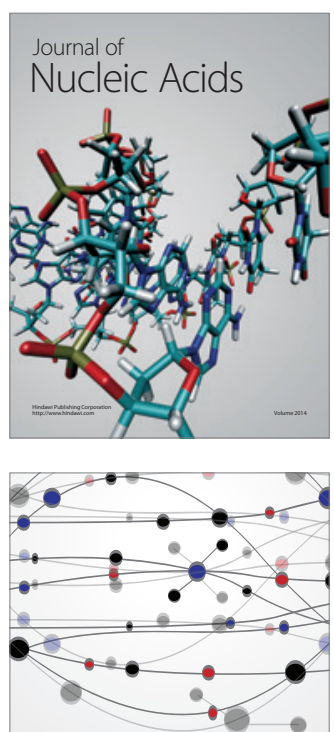

The Scientific World Journal
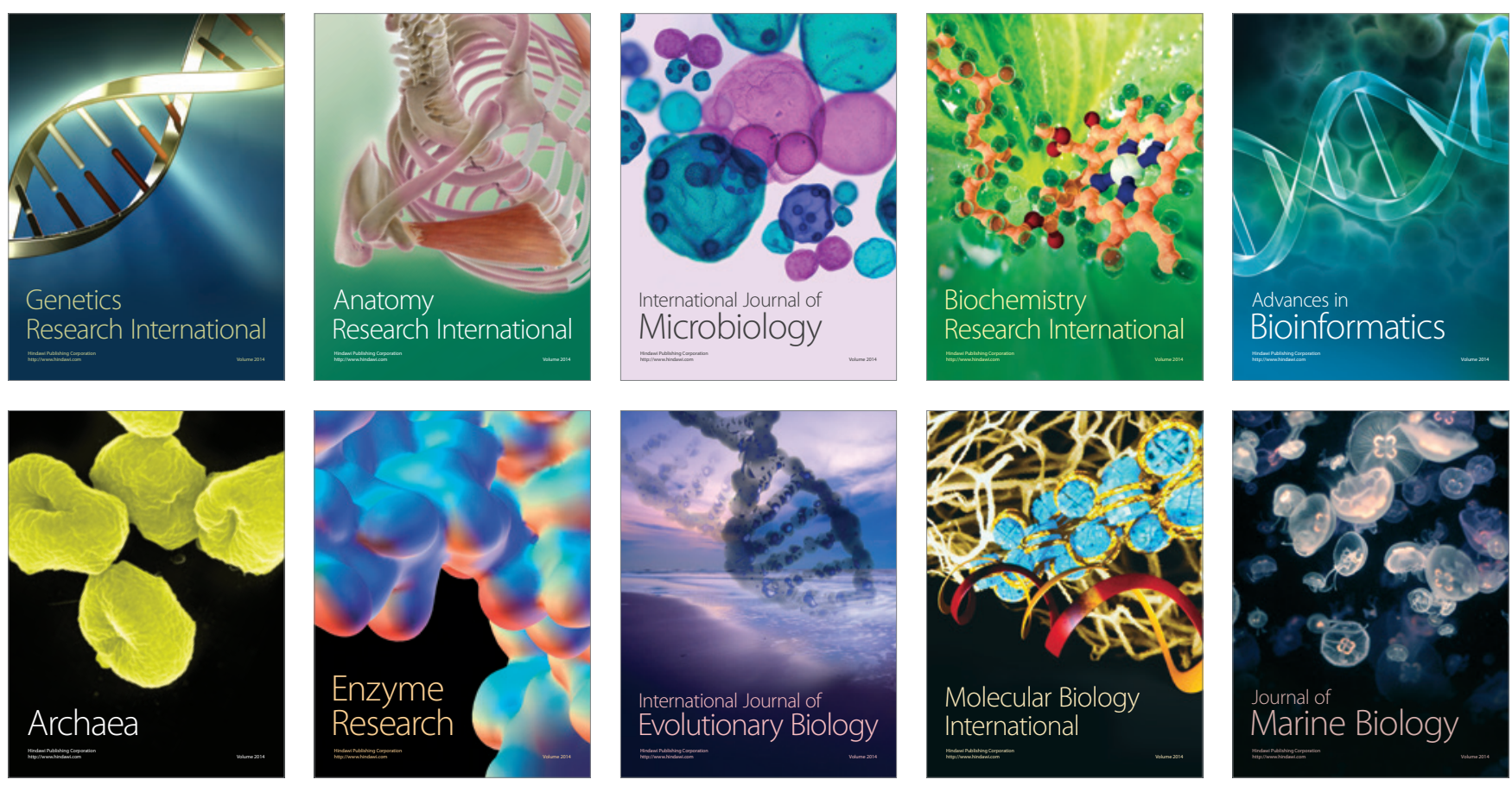